\title{
Assessment of aspiration risk in acute ischaemic stroke-evaluation of the simple swallowing provocation test
}

\author{
T Warnecke, ${ }^{1}$ I Teismann, ${ }^{1}$ W Meimann, ${ }^{2}$ S Ölenberg, ${ }^{1}$ J Zimmermann, ${ }^{1}$ C Krämer, ${ }^{1}$ \\ E B Ringelstein, ${ }^{1}$ W R Schäbitz, ${ }^{1}$ R Dziewas ${ }^{1}$
}

${ }^{1}$ Department of Neurology, University Hospital of Münster, Münster, Germany; ${ }^{2}$ Department of Neurology, Clinical Centre of Osnabrück, Osnabrück, Germany

Correspondence to:

Tobias Warnecke, MD,

Department of Neurology, University Hospital of Münster, Albert-Schweitzer-Strasse 33, 48129 Münster, Germany; Tobias.Warnecke@ukmuenster. de

Received 10 September 2007 Revised 31 October 2007 Accepted 6 November 2007

\section{ABSTRACT}

Background: Aspiration is a common complication in acute stroke patients and is strongly associated with a poor outcome. Due to an insufficient sensitivity and specificity of clinical bedside tests, further refinements are needed to improve the accuracy of clinical aspiration screening in acute stroke.

Objective: To assess the ability of the simple 2-step swallowing provocation test (SPT) to detect aspiration risk in acute stroke patients.

Methods: 100 consecutive patients with first-ever stroke were examined by SPT and fiberoptic endoscopic evaluation of swallowing (FEES) within 72 hours of stroke onset. Using FEES as an objective instrumental technique to evaluate dysphagia, statistical measures representing the ability of SPT to detect aspiration risk were calculated. Results: The incidence of endoscopically proven aspiration risk was $81 \%$. The $1^{\text {st }}$-step SPT had a sensitivity of $74.1 \%$ and a specificity of $100 \%$. Although the $2^{\text {nd }}$-step SPT showed the same $100 \%$ specificity, sensitivity was significantly lower. False-negative results of SPT appeared predominantly in subjects exhibiting leakage of liquids to pyriform sinus without a pronounced delay in swallow onset.

Conclusions: In acute stroke patients with an impairment of the pharyngeal phase of swallowing, $1^{\text {st }}$-step SPT reliably detects aspiration risk. In patients with a sole or predominant impairment of the oral phase of swallowing and a relatively intact pharyngeal phase, SPT fails to detect aspiration risk sufficiently. In the latter group, FEES or additional clinical features more specifically indicating oral-phase pathology should be considered to accurately judge the patient's aspiration risk.

Dysphagia is found in up to $78 \%$ of acute stroke patients. ${ }^{1-3}$ It is associated with aspiration pneumonia, prolonged hospital stay, increased mortality and poor long-term outcome. ${ }^{2-5}$ Several forms of clinical bedside tests depending on the ability to swallow liquids or food of various consistencies have been proposed for dysphagia assessment in acute stroke patients. ${ }^{67}$ Although these swallowing tests are safe and easily repeatable, they often have insufficient sensitivity, specificity and reliability. Hence, further refinements are needed to improve the accuracy of bedside dysphagia screening in acute stroke patients. ${ }^{7}$

Recently, Teramoto and colleagues developed a 2-step swallowing provocation test (SPT) for the detection of aspiration in elderly patients. ${ }^{8}$ As the SPT exclusively examines the involuntary swallowing reflex, it is fundamentally different from the other bedside tests. ${ }^{9}$ In our department, the SPT has become an important part of clinical dysphagia management and is also used in basic research. ${ }^{2}{ }^{10-15}$ The aim of the present study was to determine the sensitivity, specificity and predictive values of the SPT for detecting aspiration risk in acute stroke patients compared with fiberoptic endoscopic evaluation of swallowing (FEES). ${ }^{16}$

\section{PATIENTS AND METHODS}

One hundred consecutive patients with first-ever stroke were included in the study. Stroke severity was measured on admission using the National Institutes of Health Stroke Scale (NIH-SS). ${ }^{17}$ Inclusion criteria were ischaemic stroke and admittance earlier than 24 hours after symptom onset. Furthermore, in accordance with our local guidelines of stroke management, patients had to have either an NIH-SS $\geqslant 3$ points and/or had to present with a facial palsy and/or dysarthria to be eligible for advanced dysphagia assessment. Patients with a history of a preexisting dysphagia or disease probably causing dysphagia, and a severely reduced state of consciousness (ie, stupor or coma) were excluded. In all patients, the site of the brain infarction was determined by CT or MRI scans.

The SPT was carried out as described by Teramoto and colleagues in detail elsewhere. ${ }^{89}$ In brief, a thin 4-charriere catheter was inserted through the nostril into the oropharynx with its tip being placed just beneath the velum. The swallowing reflex was induced by bolus injection of $0.4 \mathrm{ml}$ (first step) and $2.0 \mathrm{ml}$ (second step) of distilled water. The onset of swallowing was identified by visual observation and simultaneous registration of the characteristic upward laryngeal movement by means of two of the examiner's fingers. The water bolus injection was administered near the end of expiration. The latent time from the beginning of water bolus injection to onset of swallowing (ie, laryngeal elevation) was measured with a stopwatch. Each of the two steps was classified as abnormal when the latent time was more than 3 seconds.

The standard FEES protocol was followed with slight modifications. ${ }^{18}$ In brief, subsequent to the anatomical/physiological assessment, the patient received teaspoon-wise three different food consistencies dyed with blue food colouring for ease of visualisation. The first food consistency introduced was pureed food, followed by liquid and soft solid food. ${ }^{19}$ For each patient, the occurrence of salient endoscopic findings was documented. The patient 
was classified as being at risk of aspiration, if aspiration itself (ie, entry of material below the true vocal folds) and/or penetration (ie, material enters the laryngeal vestibule but stays ahead of the true vocal folds) of the patient's own saliva or any feeding consistency occurred. In case of significant pharyngeal residues, the patient was observed for a further 2 minutes after the swallow to detect or exclude post-deglutitive penetration and aspiration. $^{20}$

In all subjects, SPT and FEES were performed immediately one after another within 72 hours of stroke onset. In half of the patients, the SPT was performed first; the other half received FEES at the beginning. In each patient, the examiner was blinded to the results of the other testing.

Statistical analyses were carried out with SPSS 12.0 for WINDOWS $^{\oplus}$ (SPSS Inc.). Categorical data were analysed using the $\chi 2$ test. Sensitivity $[\mathrm{TP} /(\mathrm{TP}+\mathrm{FN})]$, specificity [TN/ $(\mathrm{TN}+\mathrm{FP})]$, positive predictive value $[\mathrm{TP} /(\mathrm{TP}+\mathrm{FP})]$ and negative predictive value $[\mathrm{TN} /(\mathrm{TN}+\mathrm{FN}])$ were calculated for both steps of the SPT ( $\mathrm{TP}=$ true positive, $\mathrm{FP}=$ false positive, $\mathrm{TN}=$ true negative, $\mathrm{FN}=$ false negative).

\section{RESULTS}

Forty-six women and 54 men with a mean age of 71.43 (SD 11.81) years participated in the study. The average of NIH-SS was 9.86 (SD 4.79) points. SPT and FEES assessment could be successfully performed in all patients. Whereas patients had hemispheric strokes, 12 patients showed vertebrobasiliar strokes and 4 patients showed a combination of both.

FEES demonstrated aspiration risk in 81 patients and no aspiration risk in 19 patients. Seven patients were found without any endoscopic swallowing abnormalities. $1^{\text {st }}$-step SPT was abnormal in 60 patients, and $2^{\text {nd }}$-step SPT was abnormal in 40 patients. Table 1 summarises the sensitivities, specificities and predictive values of both steps of the SPT.

Whereas no false-positive results occurred, SPT failed to detect aspiration risk in approximately $25 \%$ of stroke patients. To gain more information about the reason for this failure, salient endoscopic findings other than penetration and aspiration of subjects with false-negative SPT results were compared with those of true negative and true positive SPT cases. Table 2 shows the frequencies of these endoscopic findings in relation to the SPT results.

In stroke patients with false-negative SPT results, the rate of leakage to the pyriform sinus was significantly higher compared with cases with a true negative SPT result $(76.2 \%$ vs. $5.3 \%$; $\mathrm{p}<0.01$ ). In comparison with subjects with a true positive SPT result, the rate of a delayed swallowing reflex was significantly lower in patients with a false-negative SPT (93.3\% vs. 19.0\%; $\mathrm{p}<0.01)$.

\section{DISCUSSION}

When compared with FEES, both steps of the SPT underestimated the aspiration risk. The $1^{\text {st }}$-step SPT failed to detect aspiration in approximately $25 \%$ of patients. The $2^{\text {nd }}$-step SPT revealed false-negative results twice as often as the $1^{\text {st }}$-step SPT, with nearly $50 \%$ normal findings despite endoscopically proven aspiration risk. Both steps of the SPT did not lead to any falsepositive result and, thereby, did not overestimate the aspiration risk. Interestingly, in 12 out of 19 patients with a true negative SPT, minor swallowing abnormalities without any penetration or aspiration were observed by using FEES. This finding indicates that minor swallowing abnormalities alone do not cause false-positive results of SPT. As a clinical consequence, there will be only very few patients in whom oral feeding and oral medication are incorrectly withheld when using SPT for decision-making of feeding strategy. As the specificity of both steps of the SPT was $100 \%$, but $1^{\text {st }}$-step SPT showed a significantly higher sensitivity in the present study, $2^{\text {nd }}$-step SPT does not gain additional information about aspiration risk, indicating that $2^{\text {nd }}$-step SPT should not be applied to acute stroke patients in clinical routine. This important difference in performance of the $1^{\text {st }}$-step SPT and the $2^{\text {nd }}$-step SPT may result from the significantly larger bolus size injected for the $2^{\text {nd }}$-step SPT

By analysing salient endoscopic findings other than penetration and aspiration, our study revealed two main subgroups of acute stroke patients to be at particular risk of aspiration. The majority $(\sim 75 \%)$ of patients with endoscopically proven aspiration risk showed a prominent disturbance of the pharyngeal phase with or without an additional dysfunction of the oral phase. Aspiration risk was associated with a pronounced disturbance of the swallowing reflex. Our study suggests SPT to be an appropriate bedside test to reliably detect aspiration risk in this particular subgroup of acute stroke patients.

Approximately $25 \%$ of acute stroke patients in our study population were at aspiration risk, because of a sole or predominant disturbance of the oral phase of swallowing. The pharyngeal phase was found to be relatively intact, and aspiration was the consequence of massive leakage to the pyriforme sinus without a significant delay in swallow onset. In this subgroup, the SPT failed to detect aspiration risk sufficiently.

However, when compared with other bedside swallowing tests, the $1^{\text {st }}$-step SPT has the advantage of a significantly higher specificity. ${ }^{7}$ As previous studies reported that videofluoroscopy or FEES are capable to detect aspiration by up to $25 \%$ more frequently compared with the clinical assessment in the same stroke population, the sensitivity of $1^{\text {st }}$-step SPT does not essentially differ from other forms of bedside examination. ${ }^{19}$ In view of the high incidence of aspiration risk in our study, the sensitivity of SPT might have been higher in a population of stroke patients similar to that of other studies in this field, exhibiting aspiration rates from $22 \%$ to $52 \%{ }^{6}{ }^{19-24}$

In conclusion, our study suggests the following approach for the initial management of dysphagia in acute stroke patients by using the $1^{\text {st }}$-step SPT:

1. Acute stroke patients with an abnormal $1^{\text {st }}$-step SPT should be given "nil by mouth".

2. Acute stroke patients with a normal $1^{\text {st }}$-step SPT, but clinical signs of an impaired oral phase of swallowing should also be kept "nil by mouth" or, if possible, should be

Table 1 Sensitivities, specificities and predictive values of SPT

\begin{tabular}{lllll}
\hline & Sensitivity (\%) & Specificity (\%) & $\begin{array}{l}\text { Positive predictive value } \\
(\%)\end{array}$ & $\begin{array}{l}\text { Negative predictive value } \\
(\%)\end{array}$ \\
\hline $1^{\text {st-step SPT }}$ & 74.1 & 100 & 100 & 47.5 \\
$2^{2^{\text {nd}}-\text { step SPT }}$ & 49.4 & 100 & 100 & 31.7 \\
\hline
\end{tabular}


Table 2 Frequencies of salient endoscopic findings other than penetration and aspiration

\begin{tabular}{llllll}
\hline Videoendoscopic findings & $\begin{array}{l}\text { SPT false } \\
\text { negative; } \mathbf{n}=\mathbf{2 1} \\
(\%)\end{array}$ & $\begin{array}{l}\text { SPT true } \\
\text { negative; } \mathbf{n = 1 9} \\
(\%)\end{array}$ & $\mathbf{p}$ Value & $\begin{array}{l}\text { SPT true } \\
\mathbf{p o s i t i v e ; ~} \mathbf{n}=\mathbf{6 0} \\
(\%)\end{array}$ & $\mathbf{p}$ Value \\
\hline Saliva pooling & $3(14.3)$ & $0(0)$ & 0.0867 & $19(31.7)$ & 0.1233 \\
Leakage to valleculae & $19(90.5)$ & $12(63.2)$ & 0.0388 & $59(98.3)$ & 0.1008 \\
Leakage to pyriform sinus & $16(76.2)$ & $1(5.3)$ & 0.0000 & $53(88.3)$ & 0.1776 \\
Residue in valleculae & $10(47.6)$ & $4(21.1)$ & 0.0786 & $35(58.3)$ & 0.3951 \\
Residue in pyriform sinus & $9(42.9)$ & $2(10.5)$ & 0.0222 & $37(61.7)$ & 0.1342 \\
Delayed swallowing reflex & $4(19.0)$ & $1(5.3)$ & 0.1880 & $56(93.3)$ & 0.0000 \\
\hline
\end{tabular}

evaluated by an objective instrumental technique (ie, FEES or videofluoroscopy in order to verify the aspiration risk).

3. In acute stroke patients with a normal $1^{\text {st }}$-step SPT and no clinical signs of oral phase swallowing disorder, oral feeding can carefully be started.

Competing interests: None declared.

\section{REFERENCES}

1. Smithard DG, O'Neill PA, Parks C, et al. Complications and outcome after acute stroke. Does dysphagia matter? Stroke 1996;27:1200-4.

2. Dziewas R, Ritter $M$, Schilling $M$, et al. Pneumonia in acute stroke patients fed by nasogastric tube. J Neurol Neurosurg Psychiatry 2004;75:852-6.

3. Martino R, Foley N, Bhogal S, et al. Dysphagia after stroke: incidence, diagnosis, and pulmonary complications. Stroke 2005;36:2756-63.

4. Mann G, Hankey GJ, Cameron D. Swallowing function after stroke: prognosis and prognostic factors at 6 months. Stroke 1999;30:744-8.

5. Smithard DG, Smeeton NC, Wolfe CD. Long-term outcome after stroke: does dysphagia matter? Age Ageing 2007;36:90-4.

6. Smithard DG, O'Neill PA, Park C, et al. Can bedside assessment reliably exclude aspiration following acute stroke? Age Ageing 1998;27:99-106.

7. Ramsay DJ, Smithard DG, Kalra L. Early assessments of dysphagia and aspiration risk in acute stroke patients. Stroke 2003;34:1252-7.

8. Teramoto $\mathbf{S}$, Matsuse $T$, Fukuchi $Y$, et al. Simple two-step swallowing provocation test for elderly patients with aspiration pneumonia. Lancet 1999;353:1243.

9. Teramoto S, Fukuchi Y. Detection of aspiration and swallowing disorder in older stroke patients: simple swallowing provocation test versus water swallowing test. Arch Phys Med Rehabil 2000;81:1517-9.

10. Dziewas R, Ludemann P, Konrad C, et al. Simple method for placing nasogastric tubes in patients with dysphagia. Lancet 2001;358:725-6.
11. Dziewas R, Schilling M, Konrad C, et al. Placing nasogastric tubes in stroke patients with dysphagia: efficiency and tolerability of the reflex placement. J Neurol Neurosurg Psychiatry 2003;74:1429-31.

12. Dziewas R, Stogbauer F, Ludemann P. Risk factors for pneumonia in patients with acute stroke. Stroke 2003:34:105.

13. Dziewas R, Soros P, Konrad C, et al. Placing nasogastric tubes and intracranial pressure. J Neurosurg Anesthesiol 2003;15:55-6.

14. Dziewas $\mathbf{R}$, Soros $P$, Ishii $R$, et al. Neuroimaging evidence for cortical involvement in the preparation and in the act of swallowing. Neuroimage 2003;20:135-44.

15. Teismann IK, Steinstraeter 0, Stoeckigt K, et al. Functional oropharyngeal sensory disruption interferes with the cortical control of swallowing. BMC Neurosci 2007;8:62.

16. Langmore SE. Evaluation of oropharyngeal dysphagia: which diagnostic tool is superior? Curr Opin Otolaryngol Head Neck Surg 2003;11:485-89.

17. Brott T, Adams HP Jr, Olinger CP, et al. Measurements of acute cerebral infarction: a clinical examination scale. Stroke 1989;20:864-70.

18. Langmore SE. Endoscopic evaluation and treatment of swallowing disorders. Thieme: New York, Stuttgart, 2001.

19. Leder SB, Espinosa JF. Aspiration risk after acute stroke: comparison of clinical examination and fiberoptic endoscopic evaluation of swallowing. Dysphagia 2002; 17:214-8.

20. Lim SH, Lieu PK, Phua SY, et al. Accuracy of bedside clinical methods compared with fiberoptic endoscopic examination of swallowing (FEES) in determining the risk of aspiration in acute stroke patients. Dysphagia 2001;16:1-6.

21. Collins MJ, Bakheit AM. Does pulse oximetry reliably detect aspiration in dysphagic stroke patients? Stroke 1997;28:1773-5

22. DePippo KL, Holas MA, Reding MJ. Validation of the 3-oz water swallow test for aspiration following stroke. Arch Neurol 1992;49:1259-61.

23. Mann G, Hankey GJ, Cameron D. Swallowing disorders following acute stroke: prevalence and diagnostic accuracy. Cerebrovasc Dis 2000;10:380-6.

24. Smith HA, Lee SH, O'Neill PA, et al. The combination of bedside swallowing assessment and oxygen saturation monitoring of swallowing in acute stroke: a safe and humane screening tool. Age Ageing 2000;29:495-9. 